\title{
Effects of caffeine on hippocampal pyramidal cells in vitro
}

\author{
R.W. Greene, H.L. Haas ${ }^{2}$ \& A. Hermann ${ }^{1}$ \\ Neurochirurgische Universitätsklinik, 8091 Zürich, Switzerland
}

1 The effects of caffeine on the electrophysiological properties of CA1 pyramidal neurones were investigated in the rat hippocampal slice preparation in vitro.

2 A concentration-dependent increase in both the extracellularly recorded excitatory postsynaptic potential (e.p.s.p.) and the population spike resulting from stimulation of the stratum radiatum could be evoked by caffeine with a threshold concentration of $10 \mu \mathrm{M}$.

3 Intracellular recordings demonstrate a caffeine-evoked decrease in resting membrane potential, an increase in input resistance, a reduction of the long afterhyperpolarization (a.h.p.) and a decrease in accommodation.

4 The interaction between caffeine and adenosine was investigated on the extracellularly recorded e.p.s.p. The maximal response evoked by caffeine was increased in the presence of adenosine and the adenosine concentration-response curve was shifted to the right in a parallel fashion in the presence of caffeine.

5 It is suggested that the effects of caffeine on hippocampal neurones may be mediated by a decrease of one or more potassium conductance(s), and that adenosine and caffeine may compete for the same electrophysiologically active receptor site on these cells.

\section{Introduction}

Caffeine, a methylated xanthine, is the most widely used central nervous system (CNS) stimulant in the world. Its behavioural effects are well known (see Goldstein et al., 1965). However, the direct effect of caffeine on the electrophysiological properties of mammalian CNS neurones is not known. Brain stem reticular formation neurones increase their firing rates following oral administration of caffeine but the mechanism for this effect is unclear (Foote, et al., 1978). In the mammalian peripheral nervous system, caffeine evokes spontaneous membrane potential hyperpolarization (Kuba \& Nishi, 1976) and potentiates the afterhyperpolarizations (a.h.ps) following action potentials of rat superior cervical ganglia neurones (Busis \& Weight, 1976). These effects are likely to be mediated by a caffeine-evoked increase in intracellular free calcium concentration, released from intracellular stores, as has been demonstrated in rat cultured dorsal root ganglia neurones (Neering \& McBurney, 1984). However, both the spontaneous hyperpolarizations

\footnotetext{
'Present address: Department of Biology, University of Konstanz, D 775 Konstanz, FRG.

${ }^{2}$ Correspondence.
}

and enhanced a.h.ps would be expected to reduce the excitability of neurones and thus are not the most likely mechanisms to mediate the caffeine-induced stimulation of the CNS. To gain some insight into the mechanism of action of caffeine, we have investigated the effects of this agent on mammalian hippocampal neurones in the in vitro slice preparation.

\section{Methods}

\section{Preparation of hippocampal slices}

Transverse slices were cut, $450 \mu \mathrm{m}$ thick, with a Mcllwain tissue chopper from the hippocampi of 24 young adult Wistar rats. The slices were transferred to the experimental chamber where they were kept completely submerged in a triple perfusion chamber modified from our original design (Haas et al., 1979). Each of the three compartments had a volume of $50 \mu \mathrm{l}$ and was independently perfusable. The solutions were maintained at $32^{\circ} \mathrm{C}$, and the flow rate was 0.3 to $0.5 \mathrm{ml} \mathrm{min}^{-1}$ in each compartment. The medium contained (in mM): $\mathrm{NaCl} 124, \mathrm{KCl} 2, \mathrm{MgSO}_{4} 1.3, \mathrm{CaCl}_{2}$ 
2.5, $\mathrm{KH}_{2} \mathrm{PO}_{4} 1.25, \mathrm{NaHCO}_{3} 26$, glucose 10 , at $\mathrm{pH} 7.4$. In some experiments tetrodotoxin (TTX) $0.003 \mathrm{mM}$ or $\mathrm{BaCl}_{2} 2 \mathrm{mM}$ was added. Sulphate and phosphate were replaced by chloride in solutions containing barium to avoid precipitations. Solutions containing drugs were usually perfused for periods of at least $10 \mathrm{~min}$ to reach plateau effects before experimental evaluation.

\section{Extracellular recording and stimulation}

A bipolar nichrome electrode was placed under visual guidance in the stratum radiatum to stimulate Schaffer collaterals and commissural fibres with monophasic $0.1 \mathrm{~ms}$ pulses of $50-150 \mu \mathrm{A}$ of current. For simultaneous recording of the resultant field excitatory postsynaptic potential (e.p.s.p.) and the population spike, the recording electrodes were placed in the stratum radiatum and stratum pyramidale, respectively (see Andersen et al., 1971), at ca. $1 \mathrm{~mm}$ distance from the stimulating electrode. The recording electrodes consisted of glass micropipettes filled with $\mathrm{NaCl}(1 \mathrm{M})$, and had resistances of 1-5 M $\Omega$. The amplitude of the e.p.s.p was measured at a fixed latency from the time of stimulation. This latency was determined before drug exposure such that the measurement occurred on the negative slope of the e.p.s.p. at about $75 \%$ of the maximum e.p.s.p. amplitude, in order to avoid contamination from the population spike.

\section{Intracellular recording}

Micropipettes for intracellular recording and current injection contained $2 \mathrm{M} \mathrm{KCl}$. Recording and current injection were performed using a high input impedance preamplifier (WPI 707). Signals were amplified, photographed from a storage oscilloscope and stored on an FM tape recorder. Membrane potential was plotted continuously on a chart recorder. Measurements, unless otherwise noted, are given as the mean \pm s.e.

\section{Drugs}

Caffeine, theophylline and adenosine were obtained from Fluka, TTX from Sigma.
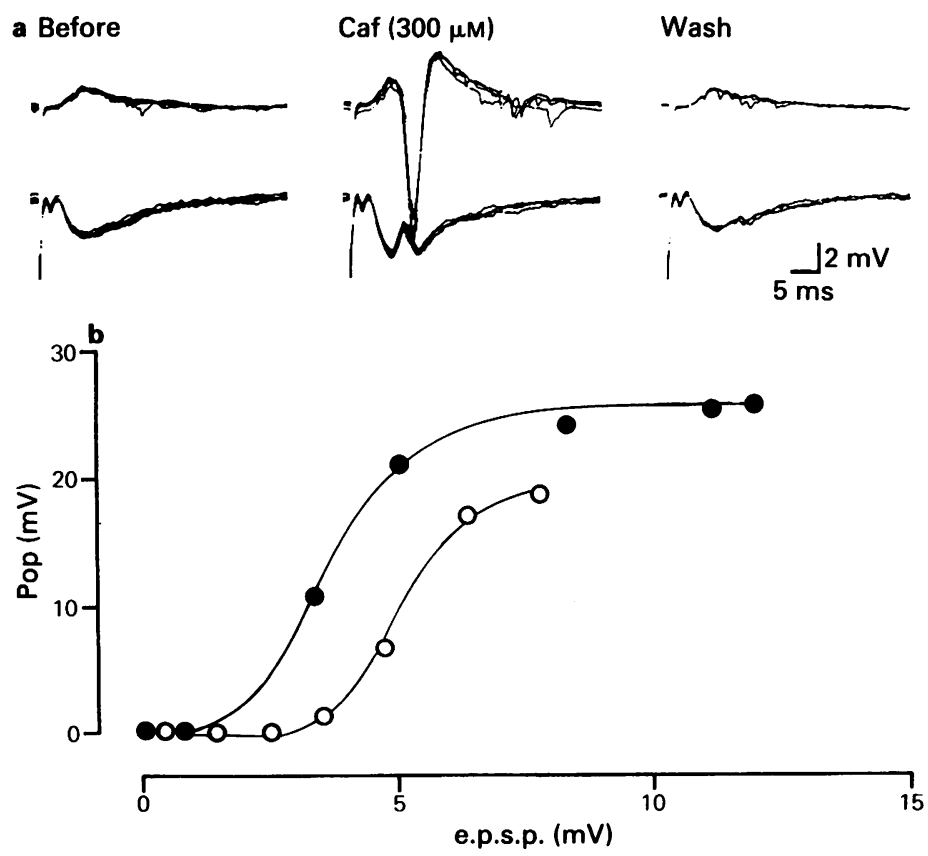

Figure 1 Effects of caffeine (Caf) on hippocampal field potentials. (a) Superimposed oscilloscope sweeps from recordings in stratum pyramidale (upper traces), and stratum radiatum (lower traces), taken before, during and after the application of caffeine $(300 \mu \mathrm{M})$. In the presence of caffeine (middle record) the e.p.s.p. was increased in amplitude and a large population spike appeared. (b) Graph of the e.p.s.p. amplitude versus the population spike amplitude obtained from the same preparation (abscissa scale: e.p.s.p. amplitude, mV; ordinate scale: population spike (Pop) amplitude, $\mathrm{mV}$ ) in the absence $(\mathrm{O})$ and presence $(O)$ of caffeine $300 \mu \mathrm{M}$. The curve was shifted to the left in the presence of caffeine. 


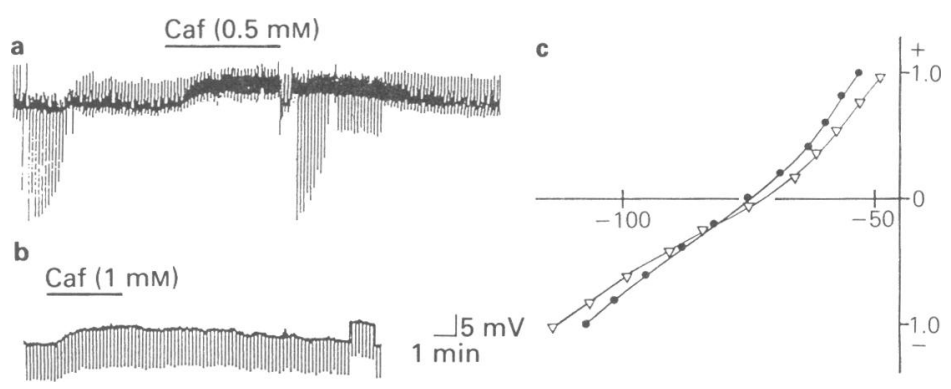

Figure 2 Effects of caffeine (Caf) on membrane potential and input resistance of hippocampal neurones. (a) Continuous recording of membrane potential before, during (horizontal bar), and after the application of caffeine $(0.5 \mathrm{mM})$. An increase in spontaneous activity is reflected in the thickened line. The large deflections are membrane potential responses to transient intracellular current injections. (b) Continuous recording of membrane potential in the presence of tetrodotoxin (TTX) before, during (horizontal bar), and after application of caffeine (1.0 mM). Calibration is the same in (a) and (b). (c) Graph of the voltage response to intracellular current injection in the presence $(\nabla)$ and absence $(O)$ of caffeine $(1.0 \mathrm{mM})$. Abscissa scale: membrane potential, $\mathrm{mV}$; ordinate scale: intracellular current injection, $\mathbf{n A}$.

\section{Results}

\section{Synaptically-evoked field potentials}

Caffeine $(10 \mu \mathrm{M}, 100 \mu \mathrm{M}, 500 \mu \mathrm{M}$ and $1 \mathrm{mM})$ and theophylline $(10 \mu \mathrm{M}$ and $100 \mu \mathrm{M})$ increased the amplitude of the extracellulary recorded e.p.s.p. and population spike of hippocampal CA 1 pyramidal cells resulting from stimulation of the stratum radiatum (Figure 1a). The input volley (i.e. the population spike of the afferent fibres) was unaffected. In 3 experiments the stratum oriens and alveus were stimulated so that an antidromic and a synaptic spike were recorded in stratum pyramidale. Only the synapticaly-evoked spike was increased by caffeine $(100 \mu \mathrm{M})$. No significant difference was found between the actions of the two methyl xanthines (Wilcoxon two-sample test, $P>0.1)$. The threshold concentration was approximately $10 \mu \mathrm{M}$, which caused a $10 \%$ increase of the e.p.s.p. amplitude. The increase evoked by $100 \mu \mathrm{M}$ caffeine was $34.8 \pm 12.9 \%(n=10)$ and that evoked by $300 \mu \mathrm{M}$ caffeine was $71.8 \pm 21.6 \%(n=10)$. The ratio of population spike amplitude to e.p.s.p. amplitude (Figure 1b) was increased by caffeine. Thus not only was the e.p.s.p. increased but a given e.p.s.p. also gave rise to a larger population spike in the presence of caffeine.

\section{Membrane potential and input resistance}

The effects of bath applied caffeine were investigated in 21 intracellularly recorded neurones with resting potentials of $72 \pm 7.6 \mathrm{mV}$. Caffeine, $100 \mu \mathrm{M}$, evoked a $4.3 \pm 1.8 \mathrm{mV}$ depolarization $(n=13)$ and a concomitant increase in input resistance of $16.6 \pm 9.3 \%$ $(n=5)$. A $1 \mathrm{mM}$ concentration of caffeine evoked a depolarization of $6.6 \pm 4.4 \mathrm{mV}(n=5)$, (Figures 2, 3a and $4 a$ ). In order to rule out caffeine-independent voltage-sensitive changes of input resistance, two procedures were employed. In the first, the input resistance was tested after the membrane potential had been returned to the level of the resting potential during the application of caffeine, by intracellular d.c. current injection. Alternatively, following the washout of caffeine and recovery of the original resting membrane potential, the neurone was artificially depolarized to the same potential as had been attained in the presence of caffeine and then input resistance was tested (Figure 2). In both cases, the increase in resistance, observed during exposure to caffeine, was found to be independent of membrane potential. Caffeine $(100 \mu \mathrm{M} ; n=9)$ also increased the amplitude and frequency of synaptically-mediated membrane potential fluctuations and the action potential (AP) firing rate.

Comparisons of the current-voltage (IV) relationships before and during the application of caffeine $(500 \mu \mathrm{M}$ and $1 \mathrm{mM})$ were made on 2 neurones as an initial step for the analysis of the ionic mechanism of the depolarization. Both of the I/V relationships in the presence of caffeine were greater than that of the control I/V curve, and in both cases the two curves intersected at $-85 \mathrm{mV}$ (Figure $2 \mathrm{c}$ ), the approximate reversal potential for potassium (Halliwell \& Adams, 1982). When caffeine was perfused onto TTX-treated hippocampal slices, its effects were similar to those on untreated slices (Figure 2b).

\section{Voltage- and calcium-sensitive conductances}

In the presence of $100 \mu \mathrm{M}$ caffeine both the long duration a.h.p. and the associated accommodation are 
a Caf $(100 \mu \mathrm{M})$

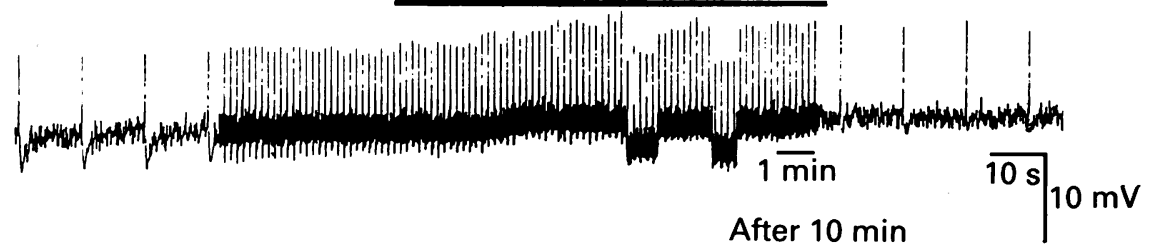

b

Caf $(100 \mu \mathrm{M})$
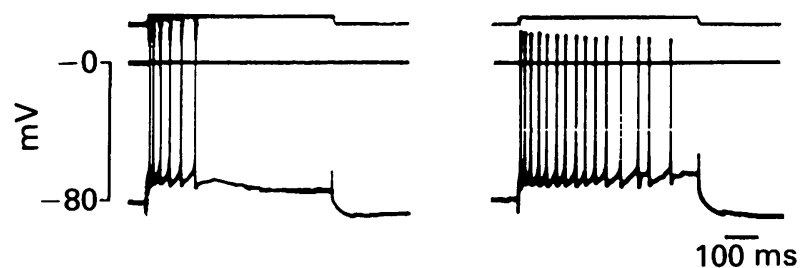

Figure 3 Effect of caffeine (Caf) on the long afterhyperpolarization (a.h.p.) and accommodation. (a) The pen recording shows a reduction in the long a.h.p. following a burst of action potentials (AP) after the addition of caffeine $(100 \mu \mathrm{M})$ to the bathing medium (an AP burst is shown by the pen recording as a single upward deflection followed by a longer duration downward deflection representing the long a.h.p.). Partial recovery is also shown. (b) Oscilloscope traces before (on the left) and during (on the right) application of caffeine (Caf; $100 \mu \mathrm{M}$ ). For each record, the top sweep reflects depolarizing intracellular current injection $(0.2 \mathrm{nA}$ during the upward deflection), the middle sweep reflects $0 \mathrm{mV}$ potential, and the bottom sweep reflects the membrane potential. During caffeine application, the membrane potential was returned to the control level by d.c. hyperpolarizing current injection and then the depolarizing current pulse was applied. Accommodation was antagonized during the caffeine application. Note the reduction of the AP amplitude.

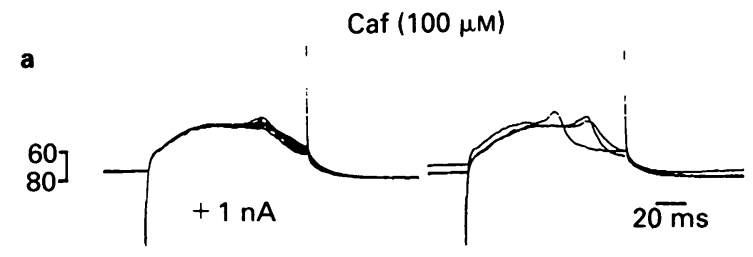

b

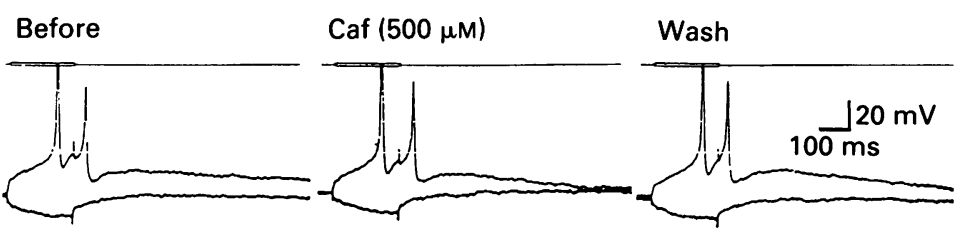

Figure 4 The effect of caffeine (Caf) on the slow action potential (AP) in tetrodotoxin (TTX)-treated slices. (a) The oscilloscope trace on the left shows the slow action potential (AP) before the addition of caffeine. The three overlapping traces on the right were made during exposure to caffeine $(100 \mu \mathrm{M})$. The trace beginning from a more depolarized level was recorded before d.c. intracellular current injection and the two traces beginning from a more hyperpolarized level were recorded after the membrane potential was returned to the control level by the current injection. (b) The addition of $\mathrm{BaCl}_{2}$ to the perfusate resulted in current evoked slow APs which appear superimposed upon a depolarizing plateau potential. Caffeine $(500 \mu \mathrm{M})$ did not alter either the slow AP amplitude or duration but did reversibly antagonize the plateau potential. 
antagonized in 5 of 7 neurones tested (Figure 3). Caffeine $(100 \mu \mathrm{M}, n=9 ; 500 \mu \mathrm{M}, n=3)$ also reduced the peak amplitude of the AP which only partially recovered in normal solution. The decrease was most prominent following the initial AP of a burst of APs (Figure $3 \mathrm{~b}$ ). The duration of the AP was unaffected by caffeine. The reduction in AP amplitude might be due to a decrease in calcium inward current which could also account for the effect of caffeine on the a.h.p. and accommodation. To examine this possibility, slices were treated with TTX to antagonize the fast inward sodium currents. The remaining high threshold, slow AP results from an interaction of depolarizing calcium current (Schwartzkroin \& Slawsky, 1977; Brown \& Griffith, 1983b) and a hyperpolarizing calcium-dependent potassium current (Schwartzkroin \& Prince, 1980; Brown \& Griffith, 1983a) and other potassium currents. This slow AP was unaffected by $100 \mu \mathrm{M}$ caffeine (Figure 4a). However, this result does not rule out effects on both the inward and outward currents.

To make a more direct investigation of the effects of caffeine on calcium currents, both TTX and $\mathrm{BaCl}_{2}$ were added to the bathing medium. $\mathrm{BaCl}_{2}$ was used because it not only passes more readily through calcium channels (Hagiwara \& Byerly, 1981), but also antagonizes many of the potassium currents that can be activated during a slow AP (Connor, 1979; Hermann \& Gorman, 1979; Constanti et al., 1981). In the TTX-barium-treated slice, small depolarizing current pulses initiated slow overshooting spikes followed by a depolarized plateau potential which lasted for several hundred milliseconds. Caffeine $(500 \mu \mathrm{M}, n=3)$ had no effect on the amplitude or duration of the slow AP. However, the depolarized plateau was reversibly reduced (Figure 4b).

\section{Interaction with adenosine}

Previous studies have indicated that methylxanthines can antagonize the binding of adenosine to its receptor (Daly et al., 1983). To test for a corresponding relationship between caffeine and adenosine with respect to electrophysiological parameters, we examined their interactions of the extracellulary recorded e.p.s.p. In 4 preparations, 24 perfusions of caffeine $(50,10,500 \mu \mathrm{M})$ resulted in a reversible and concentration-dependent antagonism of the adenosine-evoked inhibition of the e.p.s.p. Two concentration-response curves were drawn, each from a different preparation, by averaging twenty evoked e.p.s.ps for each perfusion of the drugs.

The first curve in Figure 5 shows the relationship between the log concentration of caffeine and the \% increase of e.p.s.p. Two curves were obtained, one in normal perfusion medium and the other with the addition of adenosine $(40 \mu \mathrm{M})$. Following equilibration with adenosine, the stimulation strength was increased so that the control (without caffeine) e.p.s.p. amplitude was identical to that obtained before the addition of adenosine. Under these conditions, the effect of caffeine appeared to be enhanced (Figure 5a). This was particularly evident at the concentrations of caffeine evoking the maximal response, where there was a greater than twofold increase. The second graph shows the relationship between the log-concentration of adenosine and the \% depression of the evoked e.p.s.p., in the presence and absence of $50 \mu \mathrm{M}$ caffeine (Figure 5b). The curve was shifted to the right in the presence of caffeine and the maximal response to adenosine was unaffected.
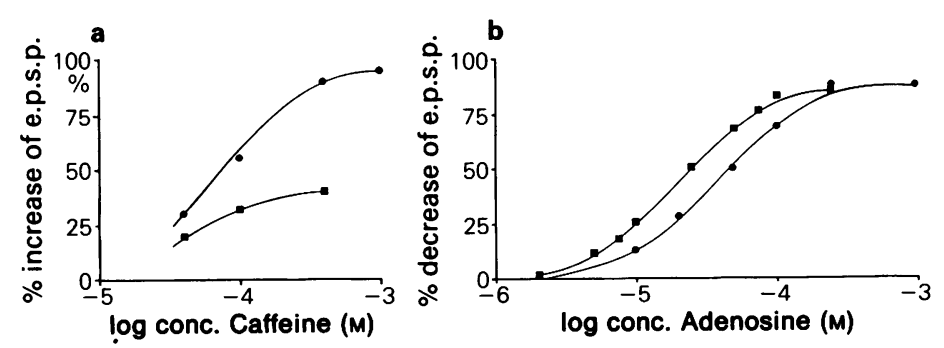

Figure 5 The interaction between caffeine ( $\mathrm{Caf}$ ) and adenosine on extracellularly recorded hippocampal e.p.s.ps. Each point in the two graphs represents 20 averaged e.p.s.ps recorded during a single perfusion. There were 7 perfusions for (a) and 15 for (b). (a) The concentration-response curve for caffeine before ( $\square$ ) and after (O) the addition of adenosine $(40 \mu \mathrm{M})$ to the bathing medium (see text). The response was measured as the $\%$ increase from base-line of the extracellularly recorded e.p.s.p. (b) Illustrates a parallel shift to the right of the adenosine concentration-response curve by caffeine $(50 \mu \mathrm{M})$; ( $(\square)$ before and $(O)$ after the addition of caffeine. The response was measured as the $\%$ reduction of the base-line extracellularly recorded e.p.s.p. 


\section{Discussion}

Our results show that caffeine excites hippocampal CA 1 neurones, as is demonstrated by its actions on both the extracellulary recorded population e.p.s.p and the population spike, and intracellulary recorded membrane responses. The excitation reflects four changes in the neuronal membrane properties: (1) a depolarization from resting membrane potential, (2) a decrease in the membrane conductance, (3) a reduction of the long duration a.h.p., and (4) a reduction of accommodation. Our data suggest that the ionic mechanism responsible for all four excitatory changes may be a decrease of one or more potassium conductance(s) (GK). The I/V curves obtained in the presence and absence of caffeine cross at the reversal potential of potassium, indicative of a reduction of GK as the mechanism of action for the first two effects. An alternative explanation could be that caffeine reduces a chloride conductance. However, this is unlikely since $\mathrm{KCl}$ filled electrodes were used which shift the chloride reversal potential $30-50 \mathrm{mV}$ in a depolarized direction (unpublished observations). It is also unlikely that a voltage-sensitive inward conductance increase is responsible for these actions of caffeine. Although such an increase might depolarize the membrane potential with an apparent increase or no change in input resistance, it would generate I/V curves that do not cross.

The long duration a.h.p. and accommodation of AP firing are mediated by an increase in GK (Gustafsson \& Wigström, 1981; Brown \& Griffith, 1983a). In both these cases the GK has been shown to be calciumdependent (Hotson \& Prince, 1980; Alger \& Nicoll, 1980; Brown \& Griffith, 1983a). Thus, the caffeineevoked decrease in the long a.h.p. and accommodation probably reflects a decrease of the calcium-dependent GK. A similar mechanism of action has been found for histamine and noradrenaline in the hippocampus (Madison \& Nicoll, 1982; Haas \& Konnerth, 1983). However, further investigation is needed to establish more firmly that the caffeine-sensitive GK is calcium-dependent.

Caffeine reduces the plateau potential but not the slow AP observed in a TTX-barium-treated preparation. The plateau potential may be generated by either a TTX-resistant increase of sodium or calcium conductance or, alternatively, a decrease of outward conductance. A similar effect of caffeine, a reduction of a slow inward current, has been demonstrated in cardiac Purkinje fibres but there too the ionic specificity is not known (Hess \& Wier, 1984). If, in hippocampal neurones, a calcium conductance is involved, then its antagonism by caffeine might contribute to the reduction of the calcium-dependent a.h.p. and accommodation.

The slow APs in TTX-barium-treated slices were unaffected by caffeine. Therefore, it is unlikely that the TTX-resistant currents are responsible for the caffeine-induced reduction of the fast AP amplitude in normal perfusion media. It is more likely that this reduction in amplitude is due to a reduced inward sodium current as has been observed in invertebrate neurones (A. Hermann, personal observation).

The four changes in neuronal membrane properties in the presence of caffeine are very different, and with respect to the long a.h.p., of opposite polarity from that seen in vertebrate peripheral (Busis \& Weight, 1976; Kuba \& Nishi, 1976) and invertebrate (Hermann, 1981) nervous systems. However, these previous studies all employed concentrations of caffeine one to two orders of magnitude greater than those used in this study, to evoke a presumed intracellular action. The effects described here probably reflect an extracellular action (see below).

Adenosine has been shown to hyperpolarize hippocampal neurones by increasing GK (Segal, 1982) and to enhance both accommodation and the long a.h.p. (Haas \& Greene, 1984). The effects of caffeine on these neurones are a remarkable mirror image of the actions of adenosine raising the possibility that exogenously applied caffeine may interact with endogenously active adenosine.

Binding studies have provided evidence for competitive inhibition of adenosine by caffeine at both the $A_{1}$ - and $A_{2}$-receptor sites (Smellie et al., 1979; Daly et $a l .$, 1983). They have further shown that an increase in the number of brain adenosine receptors follows chronic consumption of caffeine (Boulenger et al., 1983). The shift to the right of the adenosine log doseresponse curve by caffeine (similar to that seen with theophylline; Scholfield, 1978; Dunwiddie \& Hoffer, 1980 ) is consistent with a competitive antagonism of adenosine by caffeine. Such an action of caffeine could explain why the maximal potentiation of the population e.p.s.p by caffeine was further enhanced by the addition of adenosine to the perfusion medium. It follows that the maximum caffeine effect before the addition of adenosine could reflect maximal antagonism of the electrophysiological effects of endogenously active adenosine. These are an increase in resting potassium conductance and an enhancement of the a.h.p. and accommodation (Haas \& Greene, 1984).

Perhaps more relevant to the CNS stimulatory effect than the simple membrane depolarization is the modulation of neuronal responses evoked by the actions of caffeine: namely, an increase in signal to noise ratio and a potentiation of excitatory signals, especially those of long duration, which could switch the nervous system to a more responsive state. We propose that these excitatory effects are a consequence of the antagonistic effects of caffeine on adenosine present in the extracellular fluid in electrophysiologically active concentrations. 


\section{References}

ALGER, B.E. \& NICOLL, R.A. (1980). Epileptiform burst afterhyperpolarization: calcium-dependent potassium potential in hippocampal CA 1 pyramidal cells. Science, 21, $1122-1124$.

ANDERSEN, P., BLISS, T.V.P. \& SKREDE, K.K. (1971). Unit analysis of hippocampal population spikes. Exp. Brain Res., 13, 208-221.

BOULENGER, J.P., PATEL, J., POST, R.M., PARMA, A.M. \& MARANGOS, J.P. (1983). Chronic caffeine consumption increases the number of brain adenosine receptors. Life Sci., 32, 1135-1142.

BROWN, D.A. \& GRIFFITH, W.H. (1983a). Calcium-activated outward current in voltage-clamped hippocampal neurones of the guinea-pig. J. Physiol., 337, 287-301.

BROWN, D.A. \& GRIFFITH, W.H. (1983b). Persistent slow inward calcium current in voltage-clamped hippocampal neurones of the guinea-pig. J. Physiol, 337, 303-320.

BUSIS, N.A. \& WEIGHT, F.F. (1976). Spike after-hyperpolarization of a sympathetic neurone is calcium sensitive and is potentiated by theophylline. Nature, 263, 434-436.

CONNOR, J.A. (1979). Calcium current in molluscan neurones: measurement under conditions which maximize its visibility. J. Physiol., 286, 41-60.

CONSTANTI, A., ADAMS, P.R. \& BROWN, D.A. (1981). Why do barium ions imitate acetylcholine? Brain Res., 206, 244-250.

DALY, J.W., BUTTS-LAMB, P. \& PADGETT, W. (1983). Subclasses of adenosine receptors in the central nervour system: interaction with caffeine and related methylxanthines. Cell. Mol. Neurobiol., 3, 69-80.

DUNWIDDIE, T.V. \& HOFFER, B. (1980). Adenine nucleotides and synaptic transmission in the in vitro hippocampus. Br. J. Pharmac., 69, 59-68.

FOOTE, W.E., HOLMES, P., PRITCHARD, A., HATCHER, C. \& MORDES, J. (1978). Neurophysiological and pharmacodynamic studies on caffeine and on interactions between caffeine and nicotinic acid in the rat. Neuropharmacology, 17, 7-12.

GOlDSTEIN, A., KAIZER, S. \& WARREN, F. (1965). Psychotropic effects of caffeine in man. II. Altertness, psychomotor coorindation, and mood. J. Pharmac. exp. Ther., 150, 146-151.

GUSTAFSSON, B. \& WIGSTRÖM, H. (1981). Evidence for two types of afterhyperpolarization in CA 1 pyramidal cells in the hippocampus. Brain Res., 206, 462-468.

HAAS, H.L. \& GREENE, R.W. (1984). Adenosine enhances afterhyperpolarization and accommodation in hippocampal pyramidal cells. Pfluegers Arch., 402, 244-247.

HAAS, H.L. \& KONNERTH, A. (1983). Histamine and noradrenaline decrease calcium-activated potassium conductance in hippocampal pyramidal cells. Nature, 302 , $432-434$.

HAAS, H.L., SCHAERER, B. \& VOSMANSKY, M. (1979). A simple perfusion chamber for the study of nervous tissue slices in vitro. J. Neurosci. Meth., 1, 323-325.

HAGIWARA, S. \& BYERLY, L. (1981). Calcium channel. Ann. Rev. Neurosci., 4, 69-125.

HALLIWELL, J.V. \& ADAMS, P.R. (1982). Voltage-clamp analysis of muscarinic excitation in hippocampal neurons. Brain Res., 250, 71-92.

HERMANN, A. (1981). Action of caffeine on pyloric motorneurons in the crustacean stomatogastric ganglion. Comp. Biochem. Physiol., 69, 191-197.

HERMANN, A. \& GORMAN, A.L.F. (1979). Blockade of voltage-dependent and $\mathrm{Ca}^{2+}$-dependent $\mathrm{K}^{+}$current components by internal $\mathrm{Ba}^{2+}$ in molluscan pacemaker neurons. Experientia, 35, 229-231.

HESS, P. \& WEIR, W.G. (1984). Excitation-contraction coupling in cardiac Purkinje fibres. Effects of caffeine on the intracellular $\left[\mathrm{Ca}^{2+}\right]$ transient, membrane currents, and contraction. J. gen. Physiol., 83, 417-433.

HOTSON, J.R. \& PRINCE, D.A. (1980). A calcium-activated hyperpolarization follows repetitive firing in hippocampal neurones. J. Neurophysiol., 43, 409-419.

KUBA, K. \& NISHI, S. (1976). The rhythmic hyperpolarizations and the depolarization of sympathetic ganglion cells induced by caffeine. J. Neurophysiol., 39, 547-563.

MADISON, D.V. \& NICOLL, R.A. (1982). Noradrenaline blocks accommodadion of pyramidal cell discharge in the hippocampus. Nature, 299, 636-638.

NEERING, I.R. \& McBURNEY, R.N. (1984). Role for microsomal Ca storage in mammalian neurones? Nature, $309,158-160$.

SCHOLFIELD, C.N. (1978). Depression of evoked potentials in brain slices by adenosine compounds. Br. J. Pharmac., 63, 239-244.

SCHWARTZKROIN, P.A. \& PRINCE, D.A. (1980). Effects of TEA on hippocampal neurons. Brain Res., 185, 169-181.

SCHWARTZKROIN, P.A. \& SLAWSKY, M. (1977). Probable clacium spikes in hippocampal neurons. Brain Res., 135, $157-161$.

SEGAL, M. (1982). Intracellular analysis of a postsynaptic action of adenosine in the rat hippocampus. Eur. $J$. Parmac., 79, 193-199.

SMELLIE, F.W., DAVIS, C.W., DALY, J.W. \& WELLS, J.N. (1979). Alkylxanthines: inhibition of adenosine-elicited accummulation of cyclic AMP in brain slices and of brain phosphodiesterase activity. Life Sci., 24, 2475-2482. 